\title{
Diagnostic Value of Inflammatory Factors in Pathology of Bladder Cancer Patients
}

\author{
Xingxing Tang, Yudong Cao, Jia Liu, Shuo Wang, Yong Yang and Peng Du* \\ Key Laboratory of Carcinogenesis and Translational Research (Ministry of Education), Department of Urology, Peking \\ University Cancer Hospital \& Institute, Beijing, China
}

\section{OPEN ACCESS}

Edited by:

Wen G. Jiang,

Cardiff University, United Kingdom

Reviewed by:

Makoto Kobayashi,

Fukushima Medical University, Japan

Weiguang Wang,

University of Wolverhampton,

United Kingdom

*Correspondence:

Peng Du

dupeng9000@126.com

Specialty section:

This article was submitted to

Molecular Diagnostics

and Therapeutics,

a section of the journal

Frontiers in Molecular Biosciences

Received: 30 June 2020

Accepted: 08 October 2020

Published: 05 November 2020

Citation:

Tang X, Cao Y, Liu J, Wang S, Yang $Y$ and Du P (2020) Diagnostic

Value of Inflammatory Factors

in Pathology of Bladder Cancer

Patients.

Front. Mol. Biosci. 7:575483. doi: 10.3389/fmolb.2020.575483
We conducted this study to evaluate the diagnostic value of Inflammatory Factors (IFs) in the pathology of bladder cancer patients. The patients who were diagnosed with urothelial bladder carcinoma (bladder cancer) and underwent surgical treatment in our center from 2014 to 2019 were enrolled. The values of Neutrophil to Lymphocyte Ratio (NLR), derived Neutrophil to Lymphocyte Ratio (dNLR), Platelet to Lymphocyte Ratio (PLR), Lymphocyte to Monocyte Ratio (LMR), Systemic Immune-inflammation Index (SII), and Prognostic Nutritional Index (PNI) were calculated by blood routine test results before operation. After obtaining the postoperative pathology of the patients, the Area Under Curve (AUC) of Receiver Operating Characteristic (ROC) curves was calculated to evaluate the diagnostic value of these IFs in pathology and their corresponding cut-off values. A total of 641 bladder cancer patients were enrolled. The median values of NLR, dNLR, PLR, LMR, SII, and PNI were 6.33, 4.09, 156.47, 2.66, 1114.29, and 51.45, respectively. Grouped patients according to the pathological grade, the NLR, dNLR, $P L R$, and SIl of the high-grade group were significantly higher than those of the lowgrade group $(P<0.001, P<0.001, P<0.001$, and $P<0.001$, respectively), while the LMR and $\mathrm{PNI}$ were significantly lower than those of the low-grade group $(P=0.003$ and $P<0.001$ ). Divided patients into non-muscle invasion group (Tis $+\mathrm{Ta}+\mathrm{T} 1$ ) and muscle invasion group (T2 $+\mathrm{T} 3+\mathrm{T} 4)$, in which NLR, dNLR, PLR, and SIl in the muscle invasion group were significantly higher than those in the non-muscle invasion group $(P<0.001, P<0.001, P<0.001$, and $P<0.001$, respectively), while LMR and PNI were significantly lower than those in the low-grade group $(P=0.012$ and $P<0.001)$. ROC curves analyses showed that NLR, dNLR, PLR, LMR, SII, and PNI had predictive value for pathological grade $(P<0.001, P<0.001, P<0.001, P<0.001, P<0.001$, $P<0.001$, and $P<0.001$, respectively) and muscle invasion $(P<0.001, P<0.001$, $P<0.001, P<0.001, P<0.001, P<0.001$, and $P<0.001$, respectively). The results suggest the higher NLR, dNLR, PLR, SII, and lower LMR and PNI are associated with higher risk of high-grade and muscle invasive disease. However, this conclusion needs to be further clarified in the future.

Keywords: inflammatory factors, bladder cancer, neutrophil to lymphocyte ratio, derived neutrophil to lymphocyte ratio, platelet to lymphocyte ratio, lymphocyte to monocyte ratio, systemic immune-inflammation index, prognostic nutritional index 


\section{INTRODUCTION}

Blood sampling test is a routine examination for all admitted patients. White blood cell count, neutrophil count, monocyte count, lymphocyte count, platelet count and albumin concentration are common indicators in blood sampling test. Some inflammatory factors (IFs) can be easily obtained by simple calculation of these indicators. Commonly used IFs include Neutrophil to Lymphocyte Ratio (NLR), derived Neutrophil to Lymphocyte Ratio (dNLR), Platelet to Lymphocyte Ratio (PLR), Lymphocyte to Monocyte Ratio (LMR), and Systemic Immune-inflammation Index (SII) (Luo et al., 2018; Rajwa et al., 2018; Zhang et al., 2019). Currently, relevant studies have confirmed that these IFs have certain diagnostic value in different tumors (Rossi et al., 2017; Urabe et al., 2018; Losada et al., 2019). In addition, there is another IF named Prognostic Nutritional Index (PNI), which takes into account both the inflammation level and nutritional status of patients. In recent years, several studies have found that PNI has predictive and prognostic value in urothelial bladder carcinoma (bladder cancer) (Peng et al., 2017; Karsiyakali et al., 2020), which is a common tumor in urology (Siegel et al., 2017). The pathological results have a great impact on the choice of treatment and prognosis in bladder cancer patients. Therefore, exploring the examination with diagnostic value for bladder cancer pathology is of important clinical significance. At present, a number of studies have confirmed that IFs have certain diagnostic value for bladder cancer pathology (Lee et al., 2015; Rajwa et al., 2018), and our previous studies also found that NLR is associated with the risk of high-grade disease (Tang et al., 2017a,b). Considering these studies only involved some of the above IFs, we carried out this study including all these IFs to analysis their diagnostic value in the pathology of bladder cancer patients.

\section{MATERIALS AND METHODS}

\section{Study Subjects}

This single-center retrospective study included all patients diagnosed with bladder cancer in the outpatient clinic of Peking University Cancer Hospital from 2014 to 2019 and admitted for surgical treatment, including transurethral resection of bladder tumor (TURBT), partial cystectomy and total cystectomy. All enrolled patients were required to have no chemoradiotherapy before surgery and diagnosed with urothelial bladder carcinoma pathologically. Patients with other chronic inflammatory diseases or immune system diseases were excluded.

\section{Study Methods}

The basic information of patients (hospitalization number, age, gender), pathological data (tumor grade, tumor TNM stage), blood test results (white blood cell count, neutrophil count, monocyte count, lymphocyte count, platelet count, albumin content) were collected through the hospital's medical record management system. Pathological staging and histological grading of bladder cancer were based on the American Joint Commission TNM staging system on Bladder Cancer (seventh edition, 2010) (Edge and Compton, 2010). All blood sampling results were completed by the Laboratory Department of Peking University Cancer Hospital, which were the results of the first fasting blood sampling after the patient was admitted to the hospital. All pathology reports were interpreted by urologists in the Department of Pathology, Peking University Cancer Hospital. NLR was calculated as neutrophil count $\left(\times 10^{9} / \mathrm{L}\right) /$ lymphocyte count $\left(\times 10^{9} / \mathrm{L}\right)$, dNLR was calculated as neutrophil count $\left(\times 10^{9} / \mathrm{L}\right) /\left(\right.$ leukocyte count $\left(\times 10^{9} / \mathrm{L}\right)-$ neutrophil count $\left.\left(\times 10^{9} / \mathrm{L}\right)\right)$, PLR was calculated as platelet count $\left(\times 10^{9} / \mathrm{L}\right) /$ lymphocyte count $\left(\times 10^{9} / \mathrm{L}\right)$, LMR was calculated as lymphocyte count $\left(\times 10^{9} / \mathrm{L}\right) /$ monocyte count $\left(\times 10^{9} / \mathrm{L}\right)$, SII was calculated as platelet count $\left(\times 10^{9} / \mathrm{L}\right) \times$ neutrophil count $\left(\times 10^{9} / \mathrm{L}\right) /$ lymphocyte count $\left(\times 10^{9} / \mathrm{L}\right)$, and PNI was measured as albumin $(\mathrm{g} / \mathrm{L})+5 \times$ lymphocyte count $\left(\times 10^{9} / \mathrm{L}\right)$ (Luo et al., 2018; Rajwa et al., 2018; Zhang et al., 2019).

\section{Statistical Analyses}

Patients' characteristics were summarized using descriptive statistics, with categorical variables expressed as numbers and percentages and continuous variables as medians. $T$-test was used to compare continuous variables with normal distribution between groups, and chi-square test and cross-table test were used to compare categorical variables between groups. Receiver Operating Characteristic (ROC) curves were plotted to calculate the Area Under Curve (AUC) of IFs, and Youden index were calculated to select the best cut-off values. The plotting of ROC curves and the calculation of the optimal cut-off value were completed using MedCalc software (version 19), and the rest of the statistics were completed using Stata software (version 15). All tests were two-sided and $P<0.05$ was considered statistically significant.

\section{RESULTS}

\section{Patients' Characteristics}

This study eventually included 641 eligible patients with bladder cancer, with a median age of 67 years. 501 (78.16\%) patients were male patients, and more than half of the patients (52.57\%) had a history of smoking. In terms of pathological results, the number of low-grade and high-grade patients was 227 and 414, respectively. Most of the patients were diagnosed with Ta (57.57\%) and T1 (25.43\%) tumors, the other $15.91 \%$ of them were diagnosed with muscle invasive disease. The median values of NLR, dNLR, PLR, LMR, SII, and PNI were 6.33, 4.09, 156.47, 2.66, 1114.29 , and 51.45 , respectively. The patients' characteristics are shown in Table 1.

\section{Comparison of the Value of IFs Between Different Groups}

Grouped patients according to the age, gender, smoking history, pathological grade, muscle invasion, and compared whether there were significant differences between the two groups in these IFs. Grouped by age, the patients were divided into high group $(\geq 60)$ and low group $(<60)$. The dNLR of low group was significantly 
higher than that of high group $(P=0.018)$, while the PNI of high group was significantly higher than that of low group $(P<0.001)$. There were no significant differences in NLR, PLR, LMR, and SII between the two groups. In terms of gender, LMR in female patients was significantly higher than that in male patients $(P<0.001)$, and there was no significant difference in other IFs between the two groups. The PLR of smoking group was significantly lower than that of non-smoking group $(P=0.043)$, while the PNI was significantly higher than that of non-smoking group $(P=0.001)$. Grouped according to the pathological grade, the results showed that all IFs had significant differences between the two groups, in which the NLR, dNLR, PLR, and SII in the high-grade group were significantly higher than those in the low-grade group $(P<0.001, P<0.001$, $P<0.001$ and $P<0.001$, respectively), while the LMR and

TABLE 1 | Patients' characteristics.

\begin{tabular}{|c|c|}
\hline Characteristics & Total \\
\hline Patients, $\mathrm{n}$ & 641 \\
\hline Age, year, median (IQR) & $67(60-75)$ \\
\hline \multicolumn{2}{|l|}{ Sex, n (\%) } \\
\hline Male & $501(78.16)$ \\
\hline Female & $140(21.84)$ \\
\hline \multicolumn{2}{|l|}{ Smoke, n (\%) } \\
\hline No & $304(47.43)$ \\
\hline Yes & $337(52.57)$ \\
\hline \multicolumn{2}{|l|}{ Pathological grade, n (\%) } \\
\hline Low grade & $227(35.41)$ \\
\hline High grade & $414(64.59)$ \\
\hline \multicolumn{2}{|l|}{ Pathological T-stage, n (\%) } \\
\hline Tis & $7(1.09)$ \\
\hline $\mathrm{Ta}$ & $369(57.57)$ \\
\hline $\mathrm{T} 1$ & $163(25.43)$ \\
\hline T2 & $59(9.20)$ \\
\hline Т3 & $34(5.30)$ \\
\hline T4 & $9(1.40)$ \\
\hline \multicolumn{2}{|l|}{ Muscle invasion, $\mathrm{n}(\%)$} \\
\hline No (Tis $+\mathrm{Ta}+\mathrm{T} 1)$ & 539 (84.09) \\
\hline Yes $(\mathrm{T} 2+\mathrm{T} 3+\mathrm{T} 4)$ & $102(15.91)$ \\
\hline Leukocyte count, $\times 10^{9} / \mathrm{L}$, median (IQR) & $9.26(6.88-12.34)$ \\
\hline Neutrophil count, $\times 10^{9} / \mathrm{L}$, median (IQR) & $7.26(4.87-10.53)$ \\
\hline Lymphocyte count, $\times 10^{9} / \mathrm{L}$, median (IQR) & $1.24(0.87-1.70)$ \\
\hline Platelet count, $\times 10^{9} / \mathrm{L}$, median (IQR) & $198(162-243)$ \\
\hline Monocyte count, $\times 10^{9} / \mathrm{L}$, median (IQR) & $0.49(0.35-0.66)$ \\
\hline Albumin, g/L, median (IQR) & $45.50(42.80-47.50)$ \\
\hline NLR, median (IQR) & $6.33(3.14-10.94)$ \\
\hline $\mathrm{dNLR}$, median (IQR) & $4.09(2.25-6.79)$ \\
\hline PLR, median (IQR) & $156.47(120.14-225.20)$ \\
\hline LMR, median (IQR) & $2.66(1.63-3.94)$ \\
\hline SII, median (IQR) & $1114.29(626.15-2124.56)$ \\
\hline PNI, median (IQR) & $51.45(48.45-54.9)$ \\
\hline
\end{tabular}

NLR, Neutrophil to Lymphocyte Ratio; INLR, derived Neutrophil to Lymphocyte Ratio; PLR, Platelet to Lymphocyte Ratio; LMR, Lymphocyte to Monocyte Ratio; SII, Systemic Immune-inflammation Index; PNI, Prognostic Nutritional Index; IQR, Interquartile Range.
PNI were significantly lower than those in the low-grade group $(P=0.003$ and $P<0.001)$. According to whether the tumors invaded the muscle or not, the patients were divided into nonmuscle invasion group (Tis $+\mathrm{Ta}+\mathrm{T} 1$ ) and muscle invasion group $(\mathrm{T} 2+\mathrm{T} 3+\mathrm{T} 4)$. There were significant differences in all IFs between the two groups, among which NLR, dNLR, PLR, and SII in the muscle invasion group were significantly higher than those in the non-muscle invasion group $(P<0.001, P<0.001$, $P<0.001$ and $P<0.001$, respectively), while LMR and PNI were significantly lower than those in the low-grade group $(P=0.012$ and $P<0.001)$. The comparison of the values of IFs between different groups is shown in Table 2.

\section{Diagnostic Value of IFs in Pathology of Patients With Bladder Cancer}

Table 3 shows the AUC and cut-off values of the IFs for pathological grade and muscle invasion. ROC curve analyses showed that NLR, dNLR, PLR, LMR, SII, and PNI had predictive value for pathological grade (high-grade vs. low-grade) $(P<0.001, P<0.001, P<0.001, P<0.001, P<0.001, P<0.001$, and $P<0.001$, respectively), and their optimal cut-off values were $4.24,4.23,190.44,2.11,1337.80$, and 51.70, respectively, while the corresponding AUC were 0.643, 0.631, 0.579, 0.625, 0.615, and 0.672 , respectively (Figure 1). NLR, dNLR, PLR, LMR, SII, and PNI all had predictive value for muscle invasion (muscle invasion vs. non-muscle invasion) $(P<0.001, P<0.001, P<0.001$, $P<0.001, P<0.001, P<0.001$, and $P<0.001$, respectively), and their optimal cut-off values were $6.85,4.23,248.86,2.01,1723.14$, and 50.90, with corresponding AUC of 0.766, 0.744, 0.672, 0.720, 0.760 , and 0.691 , respectively (Figure 2 ).

\section{DISCUSSION}

Blood sampling test is a routine admission examination for each patient. By obtaining the white blood cell count, neutrophil count, monocyte count, lymphocyte count, platelet count and albumin concentration, it is convenient to calculate the values of NLR, dNLR, PLR, LMR, SII, and PNI. Therefore, the acquisition of the aforementioned IFs has no cost, and there is no need for patients to bear additional injuries at all. For this reason, more and more studies have begun to explore the diagnostic and prognostic value of these IFs in different tumors, and at present, meaningful results have been obtained in gastric cancer, colon cancer and breast cancer, etc. (Rossi et al., 2017; Urabe et al., 2018; Losada et al., 2019). Nowadays there is still no clear mechanism as to why these IFs can predict the tumor progression. Studies have suggested that leukocytes are indicators of systemic inflammation. When patients have infection or inflammation, leukocytes usually rise. In addition, cancer cells can also produce colony-stimulating factors to promote the increase of leukocyte counts, so the increase of leukocytes is related to tumor progression (Tachibana et al., 1995). Neutrophils may promote tumor neocapillary by releasing elastase and destroying extracellular matrix, and in turn promote tumor progression (Ginzberg et al., 2001). Monocytes can increase the number of tumor-associated macrophages in the blood, 
TABLE 2 | Comparison of the values of inflammatory factors between different groups.

\begin{tabular}{|c|c|c|c|c|c|c|c|c|c|c|c|c|c|c|c|c|c|c|c|c|c|c|c|c|c|}
\hline \multirow[t]{3}{*}{ Variables } & \multirow[t]{3}{*}{$\mathbf{N}$} & \multirow{3}{*}{ Mean } & \multicolumn{2}{|l|}{ NLR } & \multirow{3}{*}{$\begin{array}{c}P \text { - } \\
\text { value }\end{array}$} & \multicolumn{3}{|c|}{ dNLR } & \multirow{3}{*}{$\begin{array}{c}P \text { - } \\
\text { value }\end{array}$} & \multicolumn{3}{|c|}{ PLR } & \multirow{3}{*}{$\begin{array}{c}P \text { - } \\
\text { value }\end{array}$} & \multicolumn{3}{|c|}{ LMR } & \multirow{3}{*}{$\begin{array}{c}P \text { - } \\
\text { value }\end{array}$} & \multicolumn{3}{|c|}{ SII } & \multirow{3}{*}{$\begin{array}{c}P \text { - } \\
\text { value }\end{array}$} & \multicolumn{3}{|c|}{ PNI } & \multirow{3}{*}{$\begin{array}{c}P \text { - } \\
\text { value }\end{array}$} \\
\hline & & & \multicolumn{2}{|c|}{$95 \% \mathrm{Cl}$} & & \multirow[t]{2}{*}{ Mean } & \multicolumn{2}{|c|}{$95 \% \mathrm{Cl}$} & & \multirow[t]{2}{*}{ Mean } & \multicolumn{2}{|c|}{$95 \% \mathrm{Cl}$} & & \multirow[t]{2}{*}{ Mean } & \multicolumn{2}{|c|}{$95 \% \mathrm{Cl}$} & & \multirow[t]{2}{*}{ Mean } & \multicolumn{2}{|c|}{$95 \% \mathrm{Cl}$} & & \multirow[t]{2}{*}{ Mean } & \multicolumn{2}{|c|}{$95 \% \mathrm{Cl}$} & \\
\hline & & & LL & UL & & & LL & UL & & & LL & UL & & & LL & UL & & & LL & UL & & & LL & UL & \\
\hline Age, year & & & & & 0.065 & & & & 0.018 & & & & 0.557 & & & & 0.478 & & & & 0.450 & & & & $<0.001$ \\
\hline$\geq 60$ & 159 & 7.47 & 6.27 & 8.67 & & 4.44 & 3.94 & 4.94 & & 192.88 & 170.84 & 214.91 & & 3.63 & 3.20 & 4.06 & & 1691.67 & 1400.67 & 1982.67 & & 53.84 & 53.06 & 54.62 & \\
\hline$<60$ & 482 & 9.20 & 8.22 & 10.19 & & 5.34 & 4.95 & 5.74 & & 201.21 & 186.96 & 215.46 & & 3.31 & 2.81 & 3.80 & & 1870.03 & 1621.40 & 2118.65 & & 50.85 & 50.39 & 51.30 & \\
\hline Gender & & & & & 0.087 & & & & 0.175 & & & & 0.579 & & & & $<0.001$ & & & & 0.385 & & & & 0.254 \\
\hline Female & 140 & 7.46 & 6.12 & 8.80 & & 4.70 & 4.00 & 5.39 & & 205.57 & 182.30 & 228.84 & & 4.99 & 3.42 & 6.55 & & 1658.12 & 1346.72 & 1969.51 & & 51.14 & 50.18 & 52.11 & \\
\hline Male & 501 & 9.14 & 8.19 & 10.09 & & 5.24 & 4.87 & 5.60 & & 197.34 & 183.39 & 211.29 & & 2.94 & 2.72 & 3.16 & & 1872.64 & 1631.50 & 2113.78 & & 51.71 & 51.27 & 52.16 & \\
\hline Smoke & & & & & 0.756 & & & & 0.819 & & & & 0.043 & & & & 0.050 & & & & 0.254 & & & & 0.001 \\
\hline No & 304 & 8.91 & 7.56 & 10.25 & & 5.16 & 4.64 & 5.68 & & 212.15 & 192.25 & 232.05 & & 3.79 & 3.03 & 4.55 & & 1948.24 & 1583.13 & 2313.35 & & 50.90 & 50.29 & 51.51 & \\
\hline Yes & 337 & 8.65 & 7.73 & 9.57 & & 5.08 & 4.68 & 5.49 & & 187.40 & 173.31 & 201.50 & & 3.02 & 2.76 & 3.28 & & 1715.32 & 1523.08 & 1907.57 & & 52.21 & 51.68 & 52.74 & \\
\hline $\begin{array}{l}\text { Pathologica } \\
\text { grade }\end{array}$ & & & & & $<0.001$ & & & & $<0.001$ & & & & $<0.001$ & & & & 0.003 & & & & $<0.001$ & & & & $<0.001$ \\
\hline $\begin{array}{l}\text { Low } \\
\text { grade }\end{array}$ & 227 & 6.08 & 5.41 & 6.74 & & 3.94 & 3.60 & 4.29 & & 167.11 & 156.22 & 177.99 & & 4.16 & 3.19 & 5.12 & & 1249.80 & 1118.22 & 1381.39 & & 53.46 & 52.85 & 54.07 & \\
\hline $\begin{array}{l}\text { High } \\
\text { grade }\end{array}$ & 414 & 10.25 & 9.09 & 11.41 & & 5.76 & 5.31 & 6.22 & & 216.71 & 199.30 & 234.11 & & 2.96 & 2.69 & 3.23 & & 2141.60 & 1844.12 & 2439.08 & & 50.56 & 50.06 & 51.07 & \\
\hline $\begin{array}{l}\text { Muscle } \\
\text { invasion }\end{array}$ & & & & & $<0.001$ & & & & $<0.001$ & & & & $<0.001$ & & & & 0.012 & & & & $<0.001$ & & & & $<0.001$ \\
\hline $\begin{array}{c}\text { No } \\
(\text { Tis }+\mathrm{Ta}+\end{array}$ & $\begin{array}{l}539 \\
\text { 11) }\end{array}$ & 7.77 & 6.91 & 8.64 & & 4.65 & 4.31 & 5.00 & & 185.78 & 173.33 & 198.23 & & 3.60 & 3.16 & 4.04 & & 1578.21 & 1363.98 & 1792.44 & & 52.10 & 51.67 & 52.53 & \\
\hline $\begin{array}{c}\text { Yes } \\
(\mathrm{T} 2+\mathrm{T} 3+\end{array}$ & $\begin{array}{l}102 \\
T 4)\end{array}$ & 14.06 & 12.30 & 15.81 & & 7.58 & 6.79 & 8.36 & & 269.76 & 235.43 & 304.08 & & 2.26 & 1.70 & 2.81 & & 3134.04 & 2653.47 & 3614.61 & & 48.88 & 47.90 & 49.86 & \\
\hline
\end{tabular}




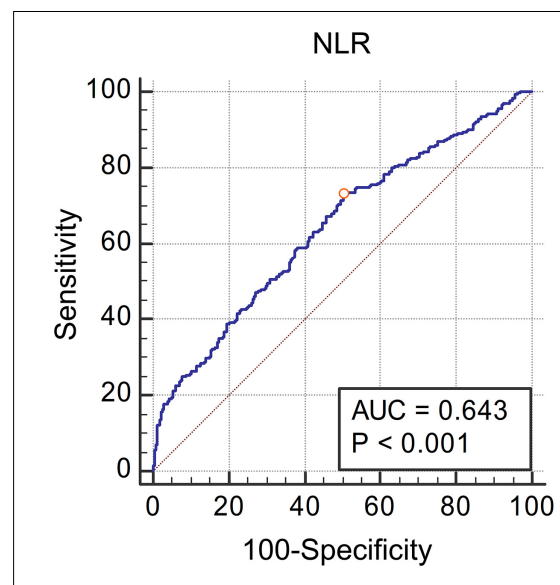

LMR

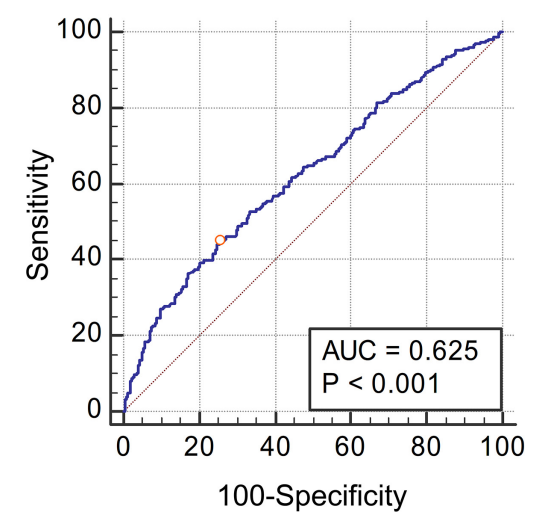

dNLR

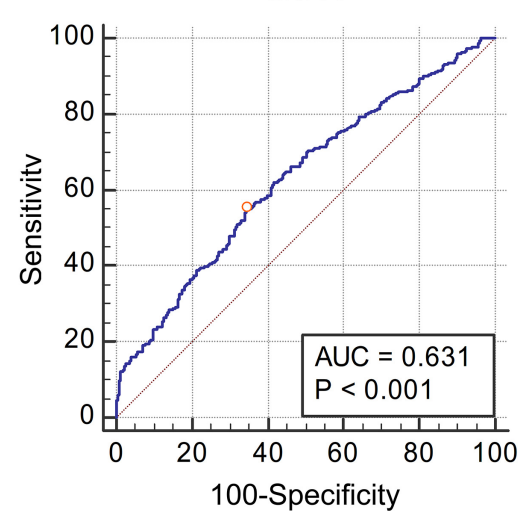

SII

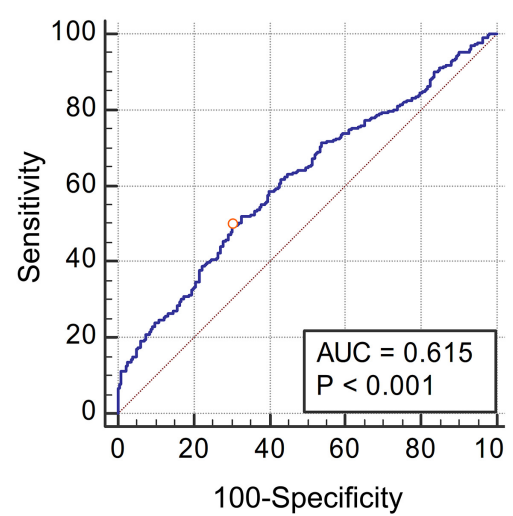

PLR

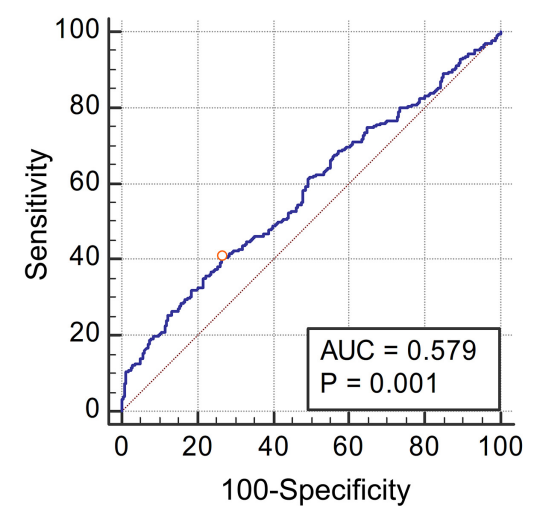

$\mathrm{PNI}$

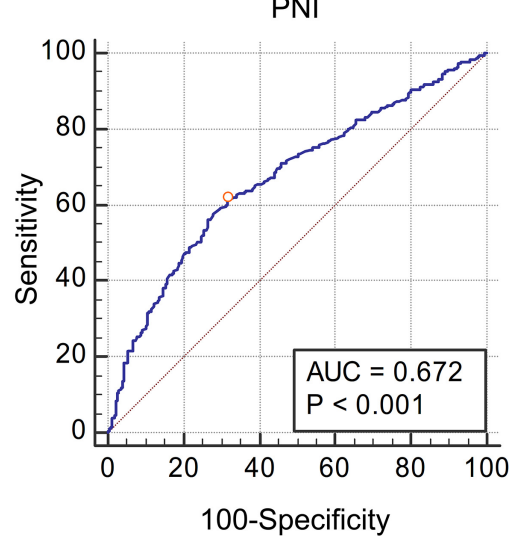

FIGURE 1 | The Receiver Operating Characteristic curves and Area Under Curve of NLR, dNLR, PLR, LMR, SII, and PNI for pathological grade. All these inflammatory factors had predictive value for pathological grade $(P<0.001, P<0.001, P<0.001, P<0.001, P<0.001$ and $P<0.001$ respectively), and the optimal cut-off values were $4.24,4.23,190.44,2.11,1337.80$, and 51.70 , respectively.

which is thought to be associated with tumor progression (Galdiero et al., 2013). Lymphocytes are related to the body's immunity, when the number of lymphocytes in tumor tissues decreases, local immunity decreases, which in turn provides an immunocompromised environment for tumor growth (Lee et al., 2012). Platelets could wrap around the tumor cells entering the circulatory system, reduce their immunogenicity, help the tumor cells to escape from immune surveillance and immune clearance, and then help the spread of tumor (Schlesinger, 2018). Albumin is the body's nutritional indicators, and low albumin usually indicates the body's malnutrition. PNI includes lymphocyte count and albumin concentration, therefore it is an index that comprehensively reflects the body's immunity and nutritional level (Karsiyakali et al., 2020).

Bladder cancer is one of the most common tumors in urology (Siegel et al., 2017), and the depth of tumor invasion will directly affect the treatment and prognosis of patients. Usually this needs to be clarified by imaging and cystoscopy, but micro muscle invasion cannot be observed by imaging, while cystoscopy is usually limited to the muscle layer and is not help for deeper invasion. Therefore, it is of great clinical significance to explore more examinations with diagnostic value in this regard. Although IFs can't replace the role of imaging and cystoscopy in the diagnosis of bladder cancer, considering IFs are simple and easy to obtain, they are meaningful even if they can only provide limited diagnostic information. Relevant studies have shown that IFs have diagnostic value for the pathology of bladder cancer (Rajwa et al., 2018; Kumarasamy et al., 2019), and our previous studies have also found that high NLR is associated with highgrade disease (Tang et al., 2017a,b). This study was further expanded by including all commonly used IFs in the analysis.

The patients were grouped according to pathological grade and muscle invasion, and the values of IFs in different groups were directly compared. The results showed that NLR, dNLR, PLR, and SII in the high-grade group were significantly higher than those in the low-grade group, while LMR and PNI were significantly lower than those in the low-grade group, suggesting that NLR, dNLR, PLR, and SII were positively correlated with pathological grade, while LMR and PNI were negatively correlated. This is consistent with the previous analysis, that is, the increase of leukocytes, neutrophils, platelets, monocytes is conducive to the growth and progression of tumor cells, while the increase of lymphocytes and albumin means stronger immunity and better nutritional status, is not conducive to the growth and progression of tumor cells, so the higher the LMR and PNI, the more unfavorable to the progression of tumor. The same 
TABLE 3 | The Area Under Curve and cut-off values of the inflammatory factors for pathological grade and muscle invasion.

\begin{tabular}{|c|c|c|c|c|c|c|c|c|c|}
\hline & \multirow[t]{2}{*}{ Cut-off value } & \multirow[t]{2}{*}{ Sensitivity } & \multirow[t]{2}{*}{ Specificity } & \multirow[t]{2}{*}{ AUC } & \multirow[t]{2}{*}{ SE } & \multicolumn{2}{|c|}{$95 \% \mathrm{Cl}$} & \multirow[t]{2}{*}{$Z$-value } & \multirow[t]{2}{*}{$P$-value } \\
\hline & & & & & & LL & UL & & \\
\hline \multicolumn{10}{|c|}{ Pathological grade } \\
\hline NLR & 4.24 & 72.71 & 49.78 & 0.643 & 0.022 & 0.604 & 0.680 & 6.423 & $<0.001$ \\
\hline$d N L R$ & 4.23 & 55.31 & 65.64 & 0.631 & 0.023 & 0.592 & 0.668 & 5.808 & $<0.001$ \\
\hline PLR & 190.44 & 40.58 & 73.57 & 0.579 & 0.023 & 0.540 & 0.618 & 3.456 & $<0.001$ \\
\hline LMR & 2.11 & 44.93 & 74.45 & 0.625 & 0.023 & 0.587 & 0.663 & 5.568 & $<0.001$ \\
\hline SII & 1337.80 & 49.76 & 69.60 & 0.615 & 0.023 & 0.576 & 0.653 & 5.095 & $<0.001$ \\
\hline PNI & 51.70 & 61.59 & 68.28 & 0.672 & 0.022 & 0.634 & 0.708 & 7.915 & $<0.001$ \\
\hline \multicolumn{10}{|c|}{ Muscle invasion } \\
\hline NLR & 6.85 & 82.35 & 61.04 & 0.766 & 0.025 & 0.731 & 0.798 & 10.525 & $<0.001$ \\
\hline$d N L R$ & 4.23 & 82.35 & 58.81 & 0.744 & 0.025 & 0.708 & 0.777 & 9.619 & $<0.001$ \\
\hline PLR & 248.86 & 45.10 & 84.97 & 0.672 & 0.031 & 0.634 & 0.709 & 5.511 & $<0.001$ \\
\hline LMR & 2.01 & 71.57 & 70.87 & 0.720 & 0.029 & 0.684 & 0.755 & 7.515 & $<0.001$ \\
\hline SII & 1723.14 & 70.59 & 74.58 & 0.760 & 0.027 & 0.725 & 0.792 & 9.747 & $<0.001$ \\
\hline $\mathrm{PNI}$ & 50.90 & 71.57 & 61.41 & 0.691 & 0.028 & 0.653 & 0.726 & 6.805 & $<0.001$ \\
\hline
\end{tabular}

NLR, Neutrophil to Lymphocyte Ratio; dNLR, derived Neutrophil to Lymphocyte Ratio; PLR, Platelet to Lymphocyte Ratio; LMR, Lymphocyte to Monocyte Ratio; SII, Systemic Immune-inflammation Index; PNI, Prognostic Nutritional Index; AUC, Area Under Curve; 95\% CI, 95\% Confidence Interval; SE, Standard Error; LL, Lower Limit; UL, Upper Limit.

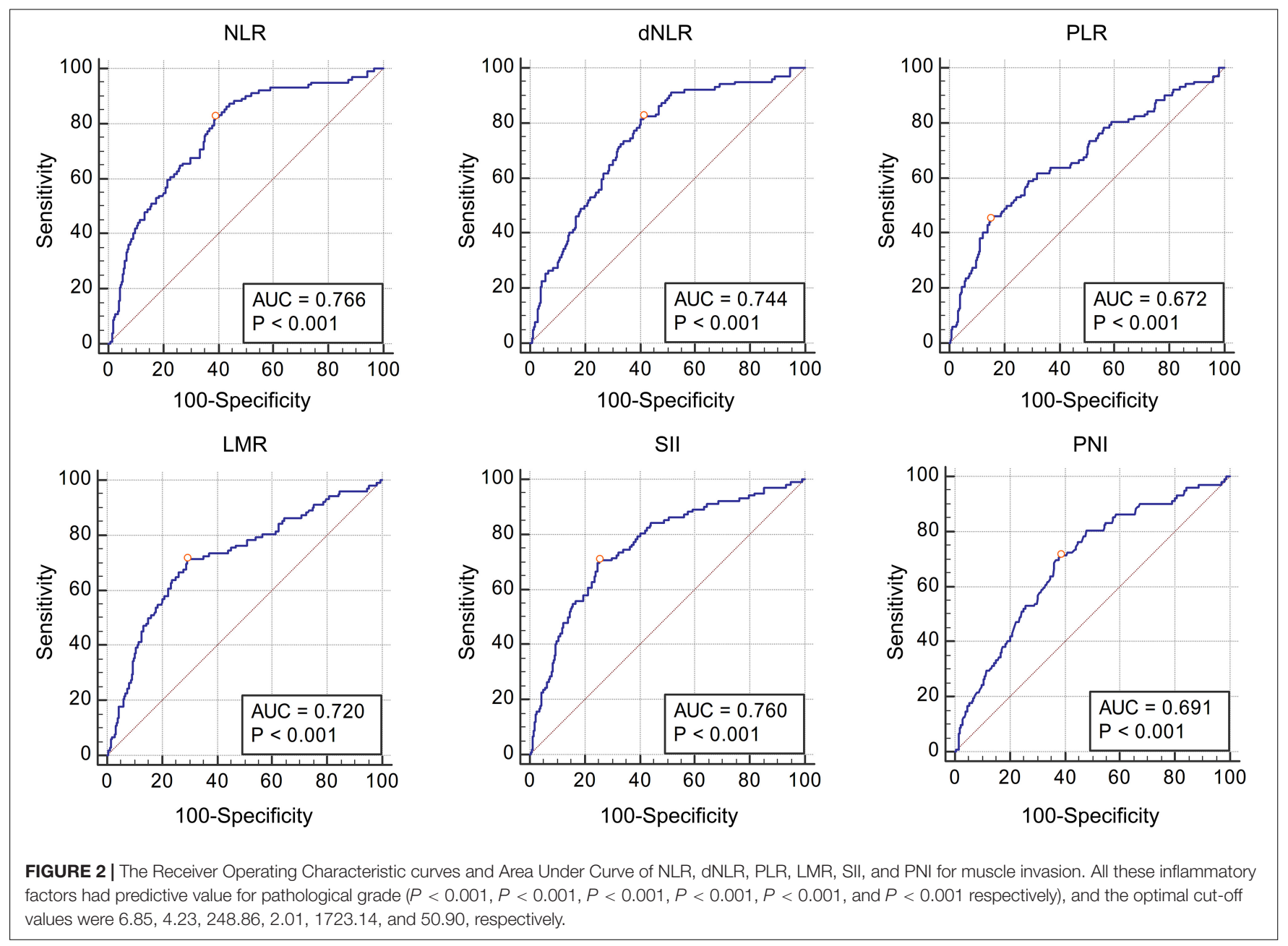


phenomenon was also observed in the muscle invasion group, where NLR, dNLR, PLR, and SII were significantly higher in the muscle invasion group than in the non-muscle invasion group, while LMR and PNI were significantly lower than in the nonmuscle invasion group. On this basis, we further analyzed the diagnostic value of IFs in bladder cancer pathology, calculated AUC by plotting ROC curves, and selected the best cut-off values. We found that NLR, dNLR, PLR, LMR, SII, and PNI had diagnostic value for pathological grade and muscle invasion. According to the definition of relevant literatures, the diagnostic value of AUC value between 0.7 and 0.8 is acceptable, AUC value between 0.6 and 0.7 is poor, and AUC value between 0.5 and 0.6 indicates no diagnostic value (Mandrekar, 2010). Although NLR, dNLR, PLR, LMR, SII, and PNI have diagnostic value for the pathological grade of bladder cancer, all the values of AUC are between 0.6 and 0.7 , suggesting these IFs only have limited diagnostic value for the pathological grade. In terms of muscle invasion, the AUC values of NLR, dNLR, LMR, and SII ranged from 0.7 to 0.8 , suggesting that these four IFs have acceptable diagnostic value for muscle invasion, while the AUC of PLR and PNI ranged from 0.6 to 0.7 , suggesting limited diagnostic value. Combined with the previous analysis, it can be concluded that the higher the NLR, dNLR, PLR, and SII, the lower the LMR and PNI, the higher the risk of high-grade and muscle invasive disease. It should be noted that for different diagnoses, the cut-off values of above IFs are different, such as NLR has diagnostic value for both pathological grade and muscle invasion, but the cut-off values are 4.24 and 6.85 , respectively. Therefore, follow-up studies with large sample sizes are needed to clarify the cut-off values of these IFs. At present, the cut-off values of these IFs in different studies are different, and clinical application of these IFs is still difficult.

In fact, several studies have similarities with our research (Cantiello et al., 2018; Rajwa et al., 2018; Ninomiya et al., 2020), but compared with these studies, our research still has many differences. The main differences between the first study (Rajwa et al., 2018) and our study are as follows. First of all, the subjects were different. That study only included patients who underwent radical cystectomy, while our study covered all patients with bladder cancer, including those who underwent TURBT, partial cystectomy and radical cystectomy. Secondly, our study is the first to analyze the diagnostic value of SII and PNI in patients with bladder cancer, which is not involved in that study. Third, due to the different population, our sample size is significantly larger. We believe that a larger sample size can provide more reliable conclusions. The second study (Cantiello et al., 2018) was the opposite of the first. Only patients with non-muscle invasive bladder cancer were included, and those with muscle invasion undergoing radical cystectomy were not included. The IFs analyzed in this study included NLR, PLR and LMR, but not dNLR, SII, and PNI. The third study (Ninomiya et al., 2020) was similar to the first study. The subjects were patients undergoing radical cystectomy. The IFs analyzed included NLR, LPR (PLR), MLR (LMR), and PNI, excluding dNLR and SII. In general, we think that compared with the above studies, the biggest difference in our study is the analysis of more IFs, especially for SII and PNI, there are very few related studies in the field of bladder cancer. In addition, we also included more patients. It is also important that the above three studies focus on the predictive value of the IFs in patients' survival, while our research focuses on the diagnostic value of the IFs for the severity of disease (pathological staging, whether tumor invades bladder muscle). The purpose of our study is to provide relevant evidence to help clinicians make a preliminary assessment of the patient's condition when they see the blood routine test results of patients considering the diagnosis of bladder cancer. In this way, when making the next diagnosis and treatment plan, it could be more targeted.

It is worth mentioning that Bladder Tumor Antigen (BTA) and Nuclear Matrix Protein 22 (NMP22) are also non-invasive tests for bladder cancer. Relevant studies have confirmed that these two tests have certain value in the diagnosis of bladder cancer (Zippe et al., 1999; Giannopoulos et al., 2001). If IFs, BTA and NMP tests are combined, the diagnostic accuracy of bladder cancer might be improved. However, although the diagnostic value of IFs in bladder cancer might be not comparable to BTA and NMP22, considering that in China and most parts of the world, most hospitals do not carry out these two tests and other similar tests due to the need for certain costs and specific equipment, the biggest advantage of IFs is low cost and easy to popularize.

One of the shortcomings of this study is its single center design. Although the sample size is enough, this might bring some bias. But on the other hand, the single center design also brings some advantages. All operations were performed by the urology department of Peking University Cancer Hospital. Blood tests and pathology were performed by the urology group of the Laboratory Department and Pathology Department of Peking University Cancer Hospital. Therefore, the quality of operation, blood tests, and pathological results are consistent, and we believe single center research also has its advantages. Another limitation of this study is that most of the included patients were non-muscle invasive patients. Considering the large prognostic gap between muscle invasive and non-muscle invasive disease, we did not analyze the prognostic value of IFs for survival, and we would conduct a separate analysis for muscle invasive bladder cancer patients after the inclusion of more patients. For the same reason, since most patients underwent TURBT and there was no information about lymph node metastasis in the pathology, we did not analyze the diagnostic value of IFs in predicting lymph node metastasis in this study, which will be analyzed separately after more patients with total cystectomy are included in the future.

\section{CONCLUSION}

Our study shows that NLR, dNLR, PLR, LMR, SII, and PNI are of diagnostic value for pathological grade and muscle invasion in patients with bladder cancer. The higher NLR, dNLR, PLR, SII, and lower LMR and PNI are associated with higher risk of highgrade and muscle invasive disease. However, this conclusion and the optimal cut-off values need to be further clarified by larger studies in the future. 


\section{DATA AVAILABILITY STATEMENT}

The raw data supporting the conclusions of this article will be made available by the authors, without undue reservation.

\section{ETHICS STATEMENT}

The studies involving human participants were reviewed and approved by the Peking University Cancer Hospital. Written informed consent for participation was not required for this study in accordance with the national legislation and the institutional requirements. Written informed consent was not obtained from the individual(s) for the publication of any potentially identifiable images or data included in this article.

\section{REFERENCES}

Cantiello, F., Russo, G. I., Vartolomei, M. D., Farhan, A. R. A., Terracciano, D., Musi, G., et al. (2018). Systemic inflammatory markers and oncologic outcomes in patients with high-risk non-muscle-invasive urothelial bladder cancer. Eur. Urol. Oncol. 1, 403-410. doi: 10.1016/j.euo.2018. 06.006

Edge, S. B., and Compton, C. C. (2010). The American Joint Committee on Cancer: the 7th edition of the AJCC cancer staging manual and the future of TNM. Ann. Surg. Oncol. 17, 1471-1474. doi: 10.1245/s10434-0100985-4

Galdiero, M. R., Bonavita, E., Barajon, I., Garlanda, C., Mantovani, A., and Jaillon, S. (2013). Tumor associated macrophages and neutrophils in cancer. Immunobiology 218, 1402-1410. doi: 10.1016/j.imbio.2013. 06.003

Giannopoulos, A., Manousakas, T., Gounari, A., Constantinides, C., Choremi-Papadopoulou, H., and Dimopoulos, C. (2001). Comparative evaluation of the diagnostic performance of the BTA stat test, NMP22 and urinary bladder cancer antigen for primary and recurrent bladder tumors. J. Urol. 166, 470-475. doi: 10.1097/00005392-20010800000015

Ginzberg, H. H., Vera, C., Qin, D., Andre, C., Mcculloch, C. A. G., Shannon, P. T., et al. (2001). Neutrophil-mediated epithelial injury during transmigration: role of elastase. Am. J. Physiol. Gastrointest. Liver Physiol. 281, G705-G717. doi: 10.1152/ajpgi.2001.281.3. G705

Karsiyakali, N., Karabay, E., and Yucetas, U. (2020). Predictive value of prognostic nutritional index on tumor stage in patients with primary bladder cancer. Arch. Esp. Urol. 73, 132-139.

Kumarasamy, C., Sabarimurugan, S., Madurantakam, R. M., Lakhotiya, K., Samiappan, S., Baxi, S., et al. (2019). Prognostic significance of blood inflammatory biomarkers NLR, PLR, and LMR in cancer-A protocol for systematic review and metaanalysis. Medicine 98:e14834. doi: 10.1097/MD.0000000000 014834

Lee, S.-M., Russell, A., and Hellawell, G. (2015). Predictive value of pretreatment inflammation-based prognostic scores (neutrophil-to-lymphocyte ratio, platelet-to-lymphocyte ratio, and lymphocyte-to-monocyte ratio) for invasive bladder carcinoma. Korean J. Urol. 56, 749-755. doi: 10.4111/kju.2015.56. 11.749

Lee, W.-C., Wu, T.-J., Chou, H.-S., Yu, M.-C., Hsu, P.-Y., Hsu, H.-Y., et al. (2012). The impact of CD4+CD25+ T cells in the tumor microenvironment of hepatocellular carcinoma. Surgery 151, 213-222. doi: 10.1016/j.surg.2011. 07.029

Losada, B., Guerra, J. A., Malón, D., Jara, C., Rodriguez, L., and Del Barco, S. (2019). Pretreatment neutrophil/lymphocyte, platelet/lymphocyte, lymphocyte/monocyte, and neutrophil/monocyte ratios and outcome in elderly breast cancer patients. Clin. Transl. Oncol. Off. Publ. Fed. Span.

\section{AUTHOR CONTRIBUTIONS}

XT contributed to prepare the manuscript and the statistical analysis. PD put forward the concept of the study and designed the study. YY reviewed the manuscript. YC, SW, and JL contributed to the data acquisition, analysis, and interpretation. XT carried out the data analysis. All authors read and approved the final manuscript.

\section{FUNDING}

The authors wish to thank the Capital's Funds for Health Improvement and Research (2018-2-1025) and Beijing Natural Science Foundation (7172044) for supporting the study.

Oncol. Soc. Natl. Cancer Inst. Mex. 21, 855-863. doi: 10.1007/s12094-0181999-9

Luo, Y., Shi, X., Li, W., Mo, L., Yang, Z., Li, X., et al. (2018). Evaluation of the clinical value of hematological parameters in patients with urothelial carcinoma of the bladder. Medicine 97:e0351. doi: 10.1097/MD.0000000000 010351

Mandrekar, J. N. (2010). Receiver operating characteristic curve in diagnostic test assessment. J. Thorac. Oncol. Off. Publ. Int. Assoc. Study Lung Cancer 5, 1315-1316. doi: 10.1097/JTO.0b013e3181 ec173d

Ninomiya, S., Kawahara, T., Miyoshi, Y., Yao, M., and Uemura, H. (2020). A retrospective study on the possible systematic inflammatory response markers to predict the prognosis of patients with bladder cancer undergoing radial cystectomy. Mol. Clin. Oncol. 13:47. doi: 10.3892/mco.2020. 2117

Peng, D., Gong, Y.-Q., Hao, H., He, Z.-S., Li, X.-S., Zhang, C.-J., et al. (2017). Preoperative prognostic nutritional index is a significant predictor of survival with bladder cancer after radical cystectomy: a retrospective study. BMC Cancer 17:391. doi: 10.1186/s12885-0173372-8

Rajwa, P., Życzkowski, M., Paradysz, A., Bujak, K., and Bryniarski, P. (2018). Evaluation of the prognostic value of LMR, PLR, NLR, and dNLR in urothelial bladder cancer patients treated with radical cystectomy. Eur. Rev. Med. Pharmacol. Sci. 22, 3027-3037. doi: 10.26355/eurrev_201805_15060

Rossi, S., Basso, M., Strippoli, A., Schinzari, G., D’Argento, E., Larocca, M., et al. (2017). Are markers of systemic inflammation good prognostic indicators in colorectal cancer? Clin. Colorectal Cancer 16, 264-274. doi: 10.1016/j.clcc.2017. 03.015

Schlesinger, M. (2018). Role of platelets and platelet receptors in cancer metastasis. J. Hematol. Oncol. J. Hematol. Oncol. 11:125. doi: 10.1186/s13045-0180669-2

Siegel, R. L., Miller, K. D., and Jemal, A. (2017). Cancer Statistics, 2017. CA Cancer J. Clin. 67, 7-30. doi: 10.3322/caac.21387

Tachibana, M., Miyakawa, A., Tazaki, H., Nakamura, K., Kubo, A., Hata, J., et al. (1995). Autocrine growth of transitional cell carcinoma of the bladder induced by granulocyte-colony stimulating factor. Cancer Res. 55, 3438-3443.

Tang, X., Du, P., and Yang, Y. (2017a). The clinical use of neutrophil-to-lymphocyte ratio in bladder cancer patients: a systematic review and meta-analysis. Int. J. Clin. Oncol. 22, 817-825. doi: 10.1007/s10147-017-1171-5

Tang, X., Wang, S., An, C., Du, P., and Yang, Y. (2017b). Preoperative high neutrophil-to-lymphocyte ratio is associated with high-grade bladder cancer. Anticancer Res. 37, 4659-4663. doi: 10.21873/anticanres. 11869

Urabe, M., Yamashita, H., Watanabe, T., and Seto, Y. (2018). Comparison of prognostic abilities among preoperative laboratory data indices in patients with resectable gastric and esophagogastric junction 
adenocarcinoma. World J. Surg. 42, 185-194. doi: 10.1007/s00268-0174146-9

Zhang, W., Wang, R., Ma, W., Wu, Y., Maskey, N., Guo, Y., et al. (2019). Systemic immune-inflammation index predicts prognosis of bladder cancer patients after radical cystectomy. Ann. Transl. Med. 7:431. doi: 10.21037/atm. 2019.09.02

Zippe, C., Pandrangi, L., and Agarwal, A. (1999). NMP22 is a sensitive, cost-effective test in patients at risk for bladder cancer. J. Urol. 161, 62-65. doi: 10.1016/s0022-5347(01) 62063-9
Conflict of Interest: The authors declare that the research was conducted in the absence of any commercial or financial relationships that could be construed as a potential conflict of interest.

Copyright $\odot 2020$ Tang, Cao, Liu, Wang, Yang and Du. This is an open-access article distributed under the terms of the Creative Commons Attribution License (CC BY). The use, distribution or reproduction in other forums is permitted, provided the original author(s) and the copyright owner(s) are credited and that the original publication in this journal is cited, in accordance with accepted academic practice. No use, distribution or reproduction is permitted which does not comply with these terms. 